1. MBBS, M.Phil, PhD.

Associate Professor Pathology

Children's Hospital \& The Institute of Child Health, Multan.

2. Ph.D

Microbiologist Pathology

Children's Hospital and

The Institute of Child Health, Multan

3. FCPS

Assistant Professor Chemical

Pathology

Combined Military Hospital Multan.

National University of Medical

Sciences, Rawalpindi.

4. MPhil, Ph.D

Scholar Microbiology

Institute of Basic Medical Sciences (IBMS),

Khyber Medical University,

Peshawar.

5. FCPS

Assistant Professor Haematology

Nishtar Medical University, Multan.

6. MD

Medical Officer Pathology

Children's Hospital and

The Institute of Child Health, Multan

Correspondence Address:

Dr. Asma Naseer Cheema

Department of Clinical Virology

Al-Jahra Hospital, Kuwait.

asma.cheema4@gmail.com

Article received on:

07/12/2020

Accepted for publication:

$15 / 02 / 2021$

\section{Curve decline of COVID-19: An analysis of first wave of disease in Pakistan- A cross-sectional study.}

\author{
Asma Naseer Cheema ${ }^{1}$, Aneeqa $\mathrm{Naz}^{2}$, Muhammad Younas ${ }^{3}$, Azra Azra ${ }^{4}$, Yasmeen Batool ${ }^{5}$, \\ Syed Zahid Ali Zaidi ${ }^{6}$
}

ABSTRACT... Objective: The objective was to observe the COVID-19 rise and fall, disease pattern, clinical presentation, effective treatment and outcome in native population. Study Design: Cross Sectional study. Setting: Children's Hospital Multan, Nishtar Hospital Multan, CMH Multan and Teaching Hospital of Kohat. Period: March 2020 to August 2020. Material \& Methods: A total of 2,186 subjects with the suspicion of COVID-19 were enrolled in this study. Chi-square test was used to see the difference in disease distribution. Results: Of total, 779 patients were found positive for COVID-19, 47.11\% were symptomatic. Of all positive subjects, no significant difference of disease spread was observed in young ( $<40$ years) versus old age ( $>40$ years) $\left[X^{2}=3.14 ; P=0.076\right)$. There were more asymptomatic female carriers than male $\left(X^{2}\right.$ $=11.68 ; P=0.001)$. The rise in cases was observed in May-June after maximum exposure on Eid festival then decline in July. Overall mortality rate was $3.98 \%$. Conclusions: The mortality rate was higher in the start, then gradually declined (9.57-0) \%. The disease prevalence was higher in male, and in the age group of 21-50 years. The mortality rate was higher in old age group (>40 years). Paracetamol and azithromycin proved to be effective in mild to moderate symptomatic patients.

Key words: $\quad$ Azithromycin, COVID-19, Paracetamol, Symptomatic.

Article Citation: Cheema AN, Naz A, Younas M, Azra A, Batool Y, Zaidi SZA. Curve decline of COVID-19: an analysis of first wave of disease in Pakistan- A crosssectional study. Professional Med J 2021; 28(6):762-771. https://doi.org/10.29309/TPMJ/2021.28.06.6257

\section{INTRODUCTION}

Coronaviruses belong to a big-cluster of positive sense ribonucleic acid (RNA)-virus family. RNAvirus emerged in the commercial hub of Wuhan, China, in December 2019. Initially, it was thought to be an anthropo-zoonotic transmission in a native wet-market of Wuhan, followed by human to human transmission that resulted in severe morbidity and mortality in humans within no time. ${ }^{1}$ The World Health Organization (WHO) entitled this emerging novel virus as severe acuterespiratory syndrome corona virus-2 (SARSCoV-2) and this particular syndrome was later termed as COVID-19. ${ }^{2}$ The current largescale outbreak of COVID-19 inflicted disastrous effects globally. So far, no definite disease pattern and treatment plan have been identified to combat this catastrophe. Data presented by different researchers revealed that morbidity and mortality pattern of COVID-19 varies widely from one population to other. Countries and territories with low population count were reported to have a bad outcome in terms of morbidity and mortality than those of higher census count. ${ }^{3}$ This suggests that other factors might be involved in the outbreak hazard rather than the population number. The actual reason behind these discrepancies is not well known. Number of hypotheses projected against the variable pattern of disease morbidity and mortality including genetic predisposition, environmental factors, dietary habits, hygiene practices and rotation of multiple strains of the virus. ${ }^{4,5}$

Alike to its erratic spread, corona exhibits extensive clinical-spectrum oscillating between asymptomatic condition to septic-shock and multiple-organ maladaptation. ${ }^{6}$ Patients present with dyspnoea (shortness of breath), which progresses to acute respiratory distress-syndrome 
(ARDS), septic shock (SS), refractory-metabolicacidosis (RMA) and disseminated intravascular coagulation (DIC). ${ }^{7}$ Until now, neither any vaccine is available to prevent SARS-CoV-2 infection nor any specific drug to treat COVID-19.8

\section{OBJECTIVE}

The objective of our study was to observe the disease pattern, clinical presentation, effective treatment strategy and outcome in native population.

\section{MATERIAL \& METHODS}

A total of 2,186 patients presenting to Children's Hospital Multan, Nishtar Hospital Multan, $\mathrm{CMH}$ Multan and Teaching Hospital of Kohat with the suspicion of COVID-19 (fever, flu like symptoms, contact history with COVID-19 patients and abroad travel history) were enrolled in this study after written informed consent from $26^{\text {th }}$ March till $14^{\text {th }}$ August 2020 . We could not follow 43 patients, so data were available for 2,143 subjects only (Figure-1). The study protocol was approved by hospital ethical committee (8920/PATH-CHM). It was a cross-sectional study. All the information of the patients regarding demographic characteristics, disease onset, initial sign and symptoms, travel history during recent past, family contact level, any associated co-morbidities and drug history were noted on a pre-designed questionnaire. Nasal/throat swab specimens taken from all suspected patients were suspended in recommended viral transport media. SARS-CoV-2 was confirmed by real time polymerase chain reaction (RT-PCR). Extraction of nucleic acids from the respiratory samples was performed with the commercialized nucleic acid extraction kits. The extracted nucleic acids were tested for SARS-CoV-2. We used Open reading frame $1 \mathrm{~b}$ to target the conserved sequence and then $\mathrm{N}$ region to target the specific sequence for SARS-CoV-2.

All admitted patients, as well as discharged patients, were followed for treatment response, any complication, disappearance of signs and symptoms/date of negative polymerase chain reaction for SARS-CoV-2. Asymptomatic patients and patients who were discharged after improvement were followed up through telephonic contact. The duration of stay in the hospital was also calculated to observe the disease burden in the hospitals. Patients were given treatment according to Government of Pakistan advisory (Table-I). Based on disease severity and treatment response, the patients were managed and treated as:

1. Asymptomatic patients who never developed symptoms (they presented to the hospital after having close contact with symptomatic COVID-19 patients), their samples were taken, they were home quarantined and followed in case they developed symptoms.

2. Mild-symptomatic patients were initially admitted, sampled for COVID-19, given treatment as mentioned in Table-l, then home quarantined and followed for disease outcome.

3. Moderate-severe symptomatic patients and the patients who had any co-morbidities remained admitted until improved and followed for disease outcome.

4. Critical patients who were put on ventilator and followed for disease outcome.

The treatment was altered according to the patient's conditions, age, any allergic reaction towards any drug and co-morbidities.

Statistical package of social sciences (SPSS) version 23.00 was used to analyze our results. The continuous variables were presented as medians and ranges. Categorical variables were described as frequencies and percentages. Chisquare test was used to see the difference in disease distribution between young versus old age and male versus female groups.

\section{RESULTS}

2,143 total participants, 1,364(63.65\%) were found negative and $779(36.35 \%)$ appeared positive for SARS-CoV-2 on RT-PCR. All positive cases were later on followed for treatment response and disease outcome. The rise in cases was observed in May and June, after maximum population exposure on Eid Festival, followed by decline in July and August. The distribution of patients who presented to the hospital with suspicion of COVID-19 is given in Figure-1. The 
average time of recovery was $11 \pm 5$ days. The dates of presentation and days of hospital stay from $26^{\text {th }}$ March until14 ${ }^{\text {th }}$ August are mentioned in Figure-2. None of the patients was admitted in the month of August. Median age (range) of the patients was 36.83 (3-74) years. The distribution of patients according to the age is given in Figure-3. Among 779 positive patients, 440 (56.48\%) were less than 40 years old, while $339(43.51 \%)$ were more than 40 years old (Table-II). Of all positive subjects, no significant difference of disease spread was observed in young ( $<40$ years) versus old age ( $>40$ years) population $\left[X^{2}=\right.$ 3.14; $P=0.076$ ). There were $72.27 \%$ male with positive polymerase chain reaction. However, there were more asymptomatic female carriers than male $\left(X^{2}=11.68 ; P=0.001\right)$ (Table-II). Of the assessed source of disease transmission, 8 (7.03\%) patients had travel history to Iran, Dubai, Indonesia, South Africa and Saudi Arabia. Thirtyseven $(32.45 \%)$ patients had known contact history with confirmed local positive symptomatic patients. Sixty-nine (60.52\%) patients had no direct contact or travel history and so were taken as unknown local transmission cases.

\section{Clinical Picture}

Based on severity of signs and symptoms, the frequency of patients is given in Table-II. The average time duration of developing respective signs and symptoms is given in Figure-4. The most prominent signs and symptoms among all the 367 patients were severe body aches, fever, loss of taste and smell, and dyspnoea (Figure-4). Patients presented with one, two and/or multiple signs and symptoms simultaneously. A large number $(75.70 \%)$ of patients presented with only respiratory complaints, followed by patients (05\%) with only gastrointestinal symptoms and without respiratory illness. We did not observe any patient in whom cardiac illness was caused solely by COVID-19.

\section{Treatment}

Treatment included paracetamol as antipyretic and was given to all 367 symptomatic patients for 5-10 days after every 4-6 hours or depending on temperature of the patient (Table-I). Oral antibiotic azithromycin was given to all symptomatic patients including children. Oral clarithromycin was given to $43(11.70 \%)$ symptomatic patients in addition to azithromycin. Meropenem injections were administered to $12(3.26 \%)$ critically sick patients. 40mg omeprazole was given to patients who were on more than one antibiotic therapy. Hydroxyl-chloroquine was also given to 17(4.63\%) critically ill patients only. Vitamin D suspension was orally administered once at the start of treatment to all symptomatic patients and injected to critically ill patients. Vitamin C tablets 500mg were given to all symptomatic patients to boost up their immune responses (Table-1). Oseltamivir was given to the patients with moderate to severe symptoms. Tramadol $(50 \mathrm{mg} / \mathrm{mL})$ injection was used in 39(10.62\%) patients. Eleven (2.99\%) patients with abnormal coagulation profile were also given anticoagulants. Supplemented oxygen was required by $53(14.4 \%)$ moderate-severe symptomatic patients.

\section{Clinical Outcome}

Overall, the average recovery time (disappearance of signs and symptoms) of patients was $11 \pm 5$ days (minimum 08 to maximum 25) days after their first presentation to the hospital or their awareness of becoming sick or of their first contact with COVID-19 symptomatic patients. Overall mortality rate was $3.98 \%$ (31/779). Mortality rate varied from March to August and was maximum in the month of May (9.5\%) (Figure-5). Mortality rate was higher in the age group of $>40$ years $[27 / 779(3.46 \%)]$ versus $<40$ years $[4 / 779(0.51 \%)]$. Of 31 total expired patients, $21(67.74 \%)$ were male. The other 30 patients were $>40$ years old,11 had hypertension and diabetes mellitus, one female patient had multinodular goitre in addition to hypertension and diabetes mellitus. The cause of death in $3(9.67 \%)$ female patients and $5(16.12 \%)$ male patients was cardiac arrest due to disseminated intravascular coagulation, while in other $23(74.19 \%)$ patients, the cause of death was respiratory failure.

The children $<12$ years old were given antibiotics according to their body weight. $B D=$ twice a day; $\mathrm{ICU}=$ intensive care unit; $\mathrm{IV}=$ intravenous; $\mathrm{OD}=$ once a day; $\mathrm{S} / \mathrm{C}=$ subcutaneous; TDS =thrice a day; GIT = gastro intestinal tract; inj = 
injection; QID = four times a day; MDI = metered dose inhaler; $A B G=$ Arterial blood gas; $R / L=$

Ringer lactate.

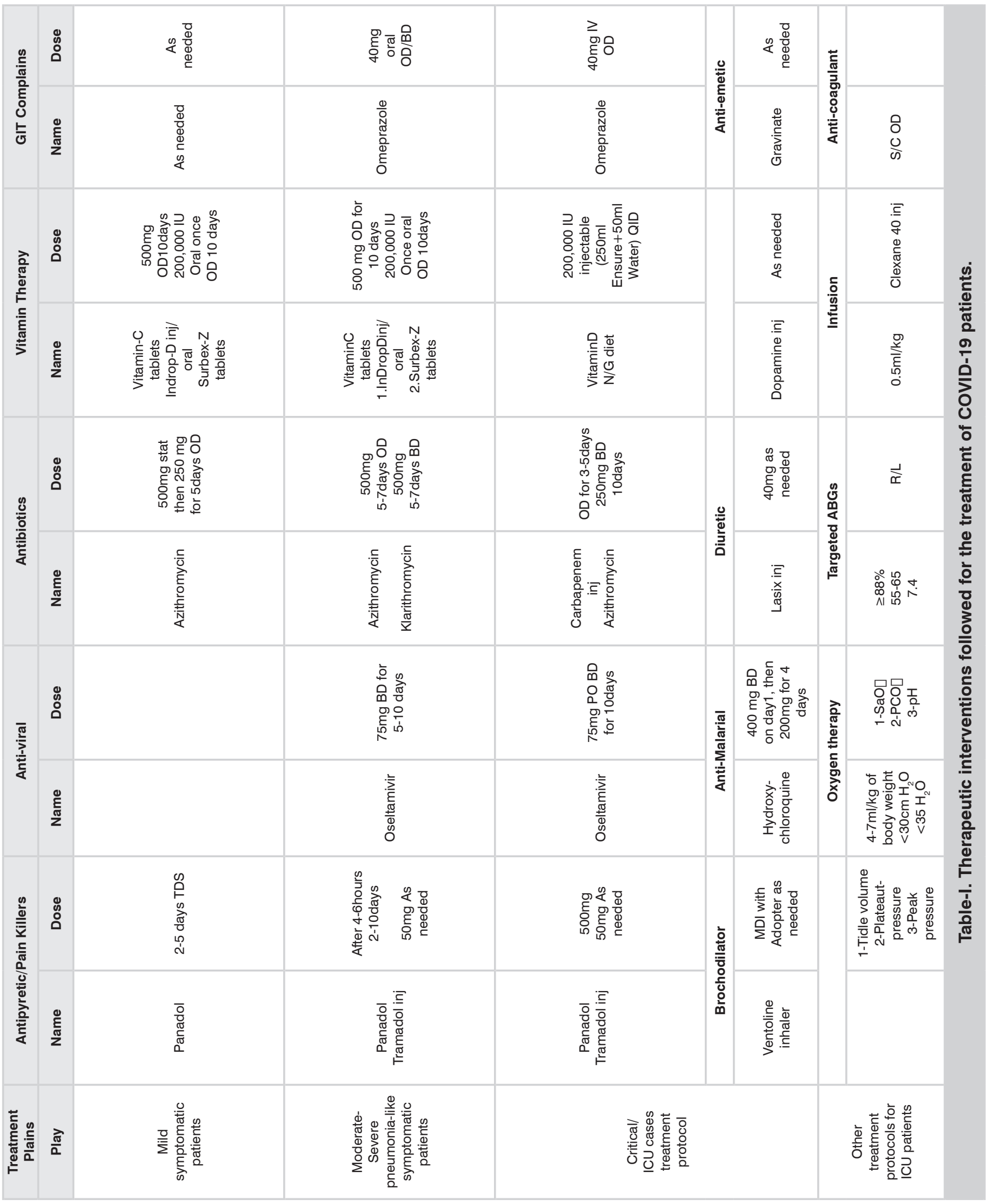




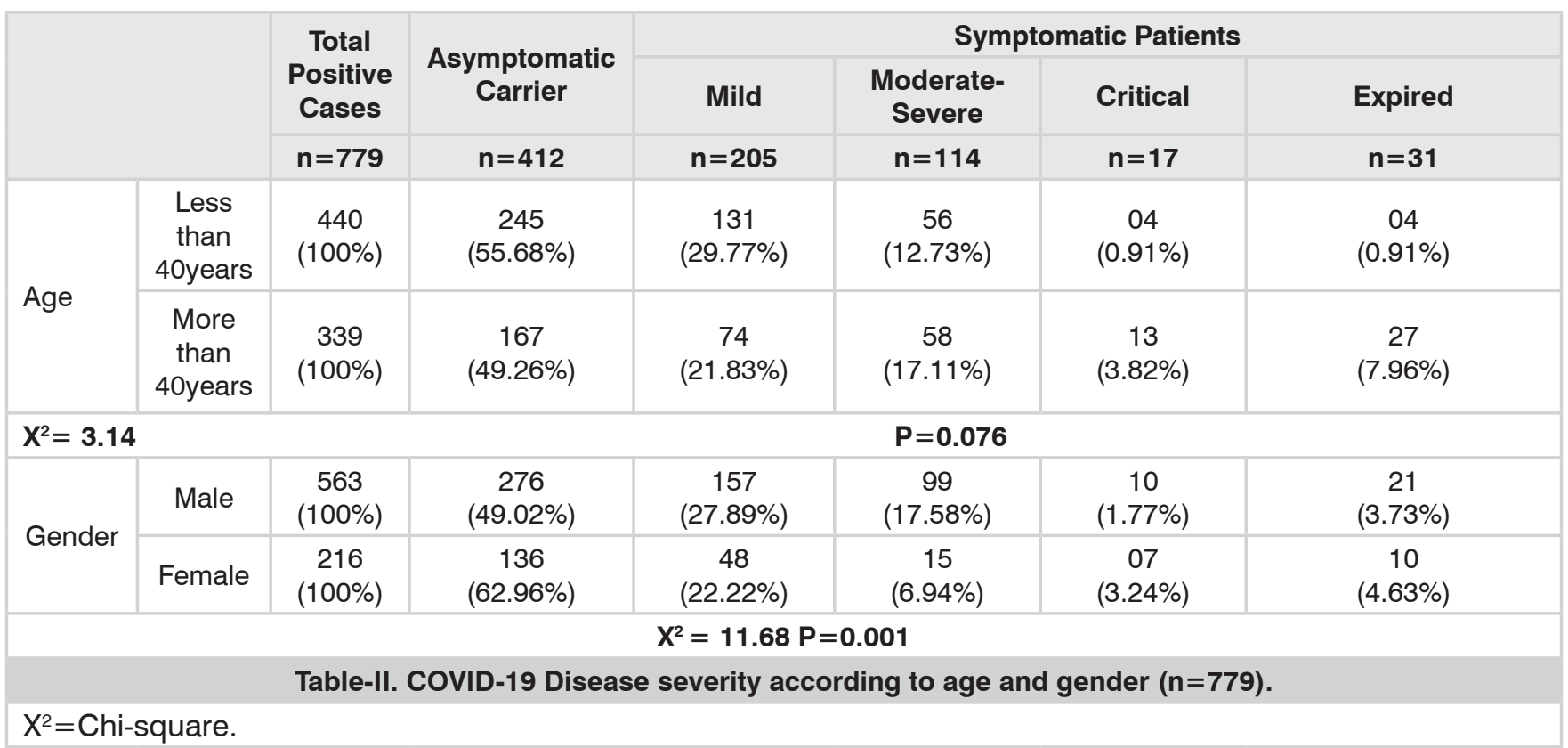

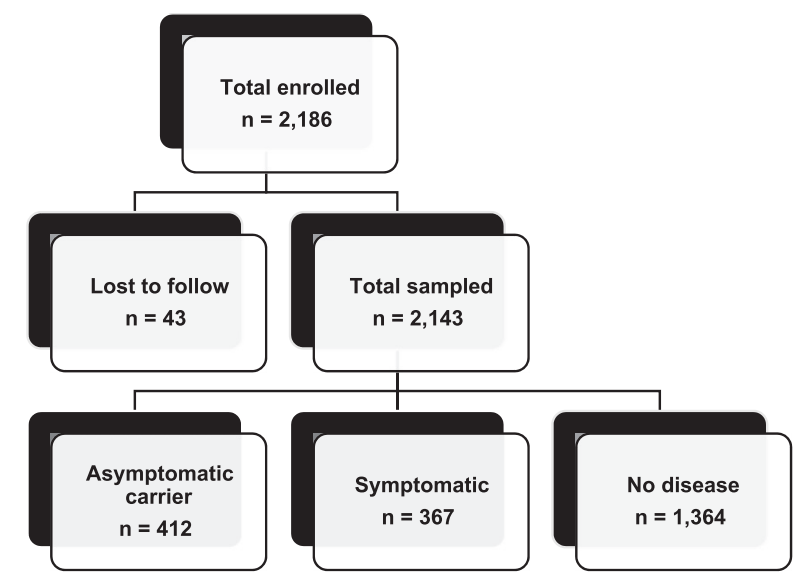

Figure-1. Study subjects who presented to the hospital with suspicion of COVID-19.

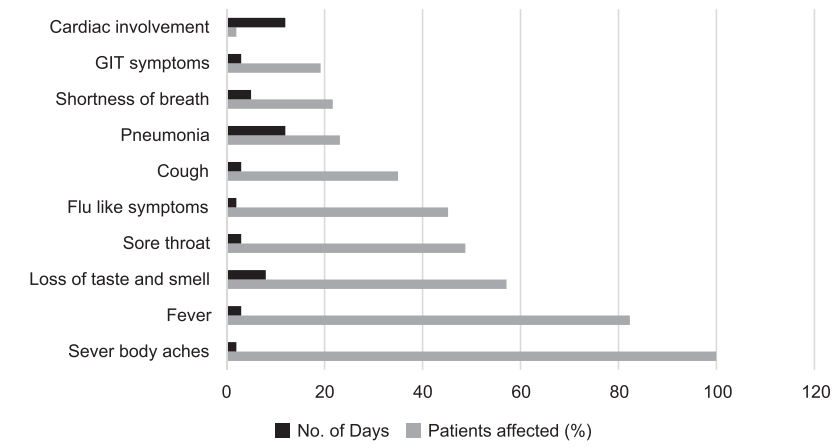

Figure-4. Onset and frequency of signs and symptoms among COVID-19 patients.

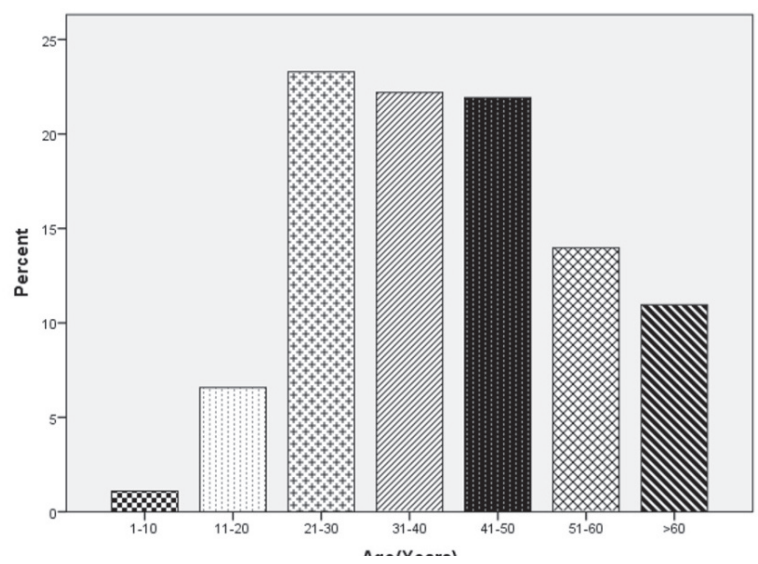

Figure-3. Distribution of COVID-19 positive cases among different age groups.

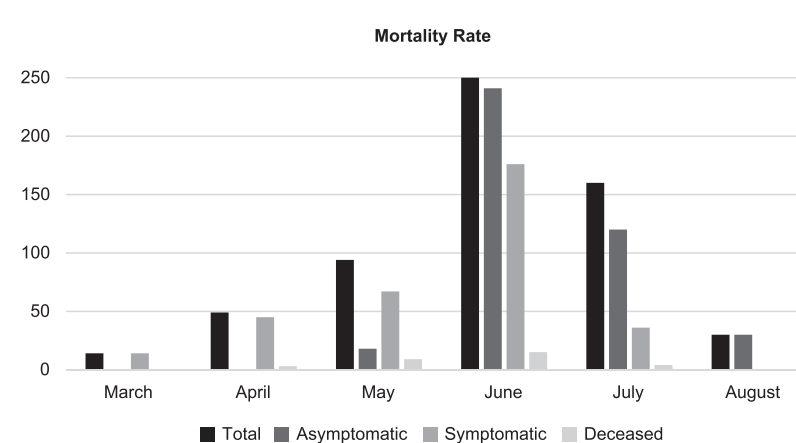

Figure-5. Month wise morbidity and mortality pattern among study subjects. 

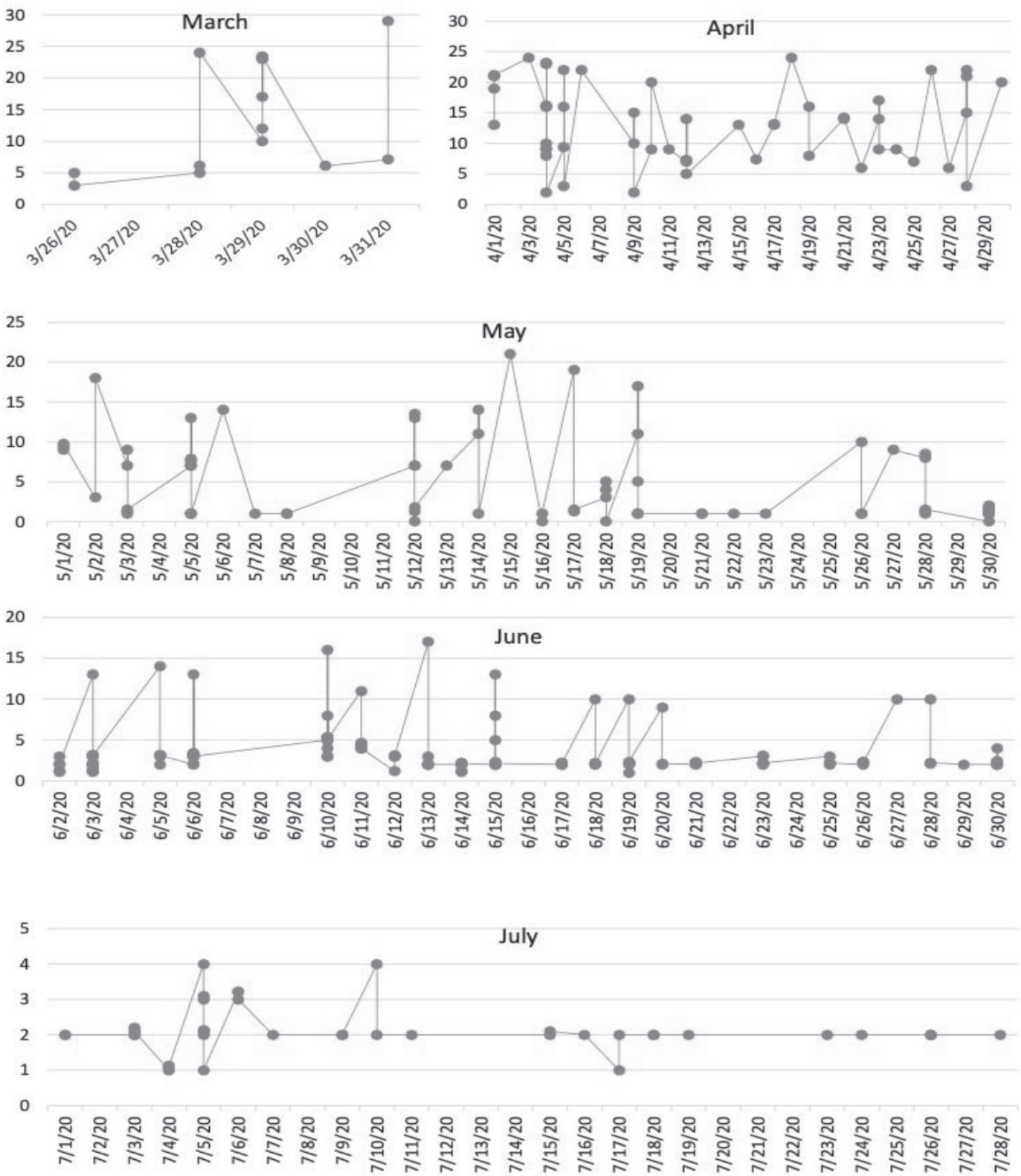

Figure-2. Dates of presentation and days of hospital stay of COVID-19 symptomatic patients from $26^{\text {th }}$ March 2020 till 14 ${ }^{\text {th }}$ August 2020.There was no admission in the month of August. (The $x$-axis is presenting the dates on which patients were admitted in the hospital. The dots are presenting the number of patients admitted on that day. When we trace the dots along $y$-axis, we can count the number of days, the patients remained admitted in the hospital. The lines joining one dot to other at $\mathrm{X}$-axis are representing the gaps in terms of days between admissions of patients). 


\section{DISCUSSION}

We followed 779 SARS-CoV-2 positive cases. All their respective clinical physiognomics, treatment response and disease outcome were regularly monitored. Most of the patients were recovered by self-isolation at homes after the treatment was prescribed by concerned hospital. Mortality rate was $3.98 \%$ in our study and overall mortality rate in Pakistan is $2.12 \%$ till to date. ${ }^{9}$ Our study data belongs to the province of Khyber Pakhtunkhwa (Pathan ethnicity) of Pakistan, where mortality rate remained quite higher $(5.2 \%)$ than other provinces. ${ }^{10}$ Overall mortality rate in Pakistan is quite comparable to our neighbouring countries like India (2.41\%) and Bangladesh (1.9\%), with whom we share genetic make-up and environmental conditions. ${ }^{11}$ In some countries mortality rate was found very high: $13.95 \%$ in Belgium, $13 \%$ in the United Kingdom and Italy and $11.3 \%$ in Netherland, while lower rates were recorded in Qatar $(0.17 \%)$, Singapore (0.2\%), United Arab Emirates (UAE) $(0.6 \%)$ and Australia (0.9\%). ${ }^{12}$ Differences in mortality may be attributed to genetic predisposition to this infection, difference in weather conditions, people's lifestyle/eating habits, living space per capita, but these myths have no scientific proof. As a matter of fact, what was actually observed is that difference in mortality in different countries is directly associated with number of infected individuals and rate of spread of infection. ${ }^{12}$ It clearly indicates that spread of infection has increased the number of deaths in line with the infection load in that region, overloaded hospitals, less accessibility of health facilities to serious patients and most importantly treatment strategies adopted by clinicians. When we evaluated the trend of infection in our study duration, maximum population fell prey to this infection after 15th May 2020 when lockdown was relaxed due to the upcoming Eid festival. We also compared our study data with overall infectivity rate in Pakistan in this time period.

The Pakistani data also showed rising tendency of infection after $15^{\text {th }}$ May 2020, with its peak incidence around the mid-June 2020, followed by continuous decline. This peak infectivity, followed by decrease in cases was in parallel to our observation of extreme local transmission cases around Eid festival, when families all over the country reunited to celebrate the occasion and maximum population exposure occurred in a short window period. Maximum exposure in short period may be attributed to the development of herd immunity and can be well related to the decline in cases of COVID-19 in Pakistan. ${ }^{13}$ However, mutant strains of the virus may initiate new cycle of infection. ${ }^{5}$ According to our findings, $56.48 \%$ patients were younger than 40 years old, while $43.52 \%$ of patients were above 40 years old. No significant difference was found in diverse age groups in terms of carrying infection. The recovery was more pronounced in younger patients as compared to old aged people having multiple health related issues and low immunity. The mortality rate was also higher in older age group. Young patients appeared to have strong immunity to protect in case they had severe illness but diagnosed timely. The old age patients seemed immunocompromised and had low sustainability against this viral infection. Beyond age structure, demographic observations shed light on noticeable gender differences in COVID-19 infectivity and mortality and that can't be ignored. We found significant difference for gender distribution of COVID-19, as $72.27 \%$ male were positive for COVID-19 in our study. The findings of male preponderance for COVID-19 infection in our study data are in accordance with the data collected from all over Pakistan. ${ }^{14}$ One of the reasons of reduced percentage of infected female in our study and overall in Pakistan may be associated with our social structure. Large percentage of our female are housewives, so they have less exposure to this infection as compared to male.

Like most of the published studies on COVID-19, we also found fever, muscle fatigue/sever body aches, loss of taste and smell as commonly occurring clinical sign and symptoms (69.29\%), but nasal congestion/runny nose were present only in few patients (21\%). ${ }^{15}$ Respiratory distress was not that worse in our population as described in other parts of the world and this could be the reason of low mortality rate in our population. Shortness of breath was comprehensively 
explained in more severe patients and inception of acute respiratory distress-syndrome is one of the symbolic features of serious COVID-19 patients that might be considered the base of its nomenclature as SARS-CoV-2. Acute respiratory distress-syndrome is considered as life intimidating because it is directly linked with lower levels of oxygen in blood and could result in multiple organ failure and leading cause of death. ${ }^{7}$ Although one third of our patients presented with respiratory illness, interestingly $5 \%$ patients solely presented with gastro-intestinal symptoms unlike the name of disease purely entitled due to absolute involvement of respiratory system. The low oxygen saturation may be attributed to low oxygen carrying capacity of haemoglobin. Dr Daniel has predicted pathogenesis of low oxygen saturation in COVID-19 patients. He states that deoxy-haemoglobin is more vulnerable to the attack of SARS-CoV-2as compared to oxyhemoglobin, which further worsens the hypoxia. ${ }^{16}$ Kiers and colleagues demonstrated the activation of platelets and coagulation system in response to low oxygen tension and inflammation. These reaction cascades cause thrombi formation throughout the body, block the blood supply to different organs and lead to respiratory failure, cardiac arrest and multiple organ failure. ${ }^{17}$

Treatments were given to the infected patients according to the severity of the disease. So far, multiple drugs are being tried for the treatment of COVID-19 patients, but all are more like supportive ones. Simple paracetamol along with azithromycin antibiotic cover worked very well for most of the symptomatic patients in our study, except for few patients who were additionally given oral clarithromycin or carbapenem injections. Hydroxychloroquine (HCQ) was also tried in critically ill patients. Some home quarantined patients (2\%) also used hydroxychloroquine on their own after getting inspired from its media told effects. Better outcomes of the combination therapy of azithromycin with hydroxychloroquine were observed in Chinese SARS-CoV-2 confirmed cases. ${ }^{18}$ Many studies also suggested the beneficial effects of hydroxychloroquine and azithromycin to avert severe cases of respiratory illness. ${ }^{19}$ Oseltamivir was also used for serious patients in our study. Oseltamivir is basically an antiviral drug used in Pakistan for the treatment of Influenza A and B infection. ${ }^{20}$ Several medicalorganizations recommended oseltamivir in patients with severe complications/high risk within 48-hours of the first signs of illness. ${ }^{21}$ We observed effective outcome of treatment given to our patients described in the Methods section (Table-I). Critically ill patients and other old aged patients were treated according to their co-morbidities along with suggested COVID-19 therapy. Many patients also tried herbal remedies and herbal medicines. As of today, there is no standard treatment strategy suggested by the World Health Organization, so patients are treated with supportive care and therapy and according to severity of the disease. We also used vitamin C and vitamin $D$ in our patients as a part of treatment strategy. A study published in the United States of America showed the beneficial effects of vitamin C, in which 167-patients with sepsis-related acute respiratory distress-syndrome were administered vitamin C (15g/day for 4days). ${ }^{22,23}$ Numerous published data also described the role of vitamin $D$ in regulation of healthy immunity. ${ }^{24}$ Vitamin $D$ has prominent function to affect immune cells, by inhibiting B lymphocyte self-division, blocking $B$ lymphocytes cellular differentiation and antibodiesexcretion. ${ }^{25,26,27}$

The world population infection trend showed that $40-45 \%$ percent of individuals infected by SARSCoV-2 were asymptomatic healthy-carriers and had more potential to spread disease and this is great matter of concern because these carrier patients can transmit viral infection to the healthy people for extended time period than the usual 14 days. We also found $52.89 \%$ healthy carriers in our study group. The number of healthy carriers is comparable with other populations and this reveals that disease severity certainly relates to personal physiology/genetic make-up and that needs to be worked-up. ${ }^{28}$

To our knowledge, this is the first study in the local population that quantified the disease pattern in terms of its spread, therapeutic effectiveness and clinical outcome. This study provides inclusive 
information of disease distribution across age/ gender, frequency of morbidity/mortality and related clinical manifestation of COVID-19 in native population.

Though, we collected the data from sufficient number of people but only from two regions of Pakistan. The larger data involving more regional areas could have given more valuable findings. We could not determine the route of transmission of disease spread whether it is widely spread through respiratory droplets or orofecal route. We also could not confirm the anthropozoonotic transmission of SARS-CoV-2as it was determined in commercial city of Wuhan, China. No potential animal reservoirs of infection have yet been identified in Pakistan for SARS-CoV-2. Research protocols and organized data collection tools should be arranged, scrutinized, approved and disseminated across the globe to implement the research outcome on timely manner and close the knowledge gaps against this novel virus.

\section{CONCLUSION}

The mortality rate was higher in the start then gradually declined (maximum, 9.57\% and minimum 0\%). The disease prevalence was higher in male, and in the age group of 21-50 years. The mortality rate was higher in old age group ( $>40$ years). Paracetamol and azithromycin proved to be effective in mild to moderate symptomatic patients.

\section{Copyright@ 15 Feb, 2021.}

\section{REFERENCES}

1. Li Q, Guan X, Wu P, Wang X, Zhou L, Tong Yet al. Early transmission dynamics in Wuhan, China, of novel coronavirus-infected pneumonia. N Engl J Med. 2020; 382(13):1199-1207.

2. World Health Organization. Global research on coronavirus disease (COVID-19). Accessed in 2020 (Nov 11).

3. Anderson RM, Heesterbeek $H$, Klinkenberg D, Hollingsworth TD. How will country-based mitigation measures influence the course of the COVID-19 epidemic? Lancet. 2020; 395(10228):931-4.

4. Yao H, Lu X, Chen Q, Kaijin Xu, Yu Chen, Linfang Cheng et al. Patient-derived mutations impact pathogenicity of SARS-CoV-2.MedRxiv. 2020.
5. Callaway E. The corona virus is mutating- does it matter? Nature. Available Accessed in 2020 (Nov 11).

6. Cascella M,Rajnik M, Cuomo A, Dulebohn SC, Napoli RD. Features, Evaluation and Treatment Coronavirus. 2020. In: Stat Pearls [Internet].Treasure Island (FL): Stat Pearls Publishing. PMID: 32150360.

7. Guan WJ, Ni ZY, Hu Y, Liang WH, Ou CQ, He JX et al. Clinical characteristics of coronavirus disease 2019 in China. New EnglJMed. 2020; 382(18):1708-20.

8. Lu H. Drug treatment options for the 2019-new coronavirus (2019-nCoV). BiosciTrends.2020;14(1):69-71.PMID:31996494.

9. Government of Pakistan. COVID-19 Situation. Available from: https://covid.gov.pk. Accessed in 2020 (Nov 11).

10. Yusufzai A. Peshawar records highest mortality rate. DAWN. Available from: https://www.dawn.com/ news/1552545. Accessed in 2020 (Nov 11).

11. World Health Organization. Who Coronavirus Disease (COVID-19) Dashboard. Accessed in 2020 (Nov 11).

12. Noor AU, Maqbool F, Bhatti ZA, Khan AU. Epidemiology of CoViD-19 Pandemic: Recovery and mortality ratio around the globe. Pak J Med Sci.2020; 36(COVID19S4):S79-84.

13. Government of Pakistan. Pakistan cases details. Available from: http://covid.gov.pk/stats/pakistan. Accessed in 2020 (Nov 11).

14. Abid K, Bari YA, Younas M, Tahir Javaid S, Imran A. Progress of COVID-19 Epidemic in Pakistan. Asia Pac J Public Health. 2020; 32(4):154-6.

15. Chen N, Zhou M, Dong X, Qu J, Gong F, Han Y, et al. Epidemiological and clinical characteristics of 99 cases of 2019 novel coronavirus pneumonia in Wuhan, China: A descriptive study. Lancet. 2020; 395(10223):507-13.

16. Daniel $\mathrm{Y}$, Hunt BJ, Retter A, Henderson K, Wilson $S$, Sharpe $\mathrm{CC}$ et al. Haemoglobin oxygen affinity in patients with severe COVID-19 infection. $\mathrm{Br} J$ Haematol. 2020; 190(3): e126-7.

17. Kiers D, Tunjungputri RN, Borkus R, Scheffer GJ, de groot PG, Urbanus RT, et al. The influence of hypoxia on platelet function and plasmatic coagulation during systemic inflammation in humans in vivo. Platelets. 2019; 30(7):927-30. 
18. Gautret P, Lagier JC, Parola P, Meddeb L, Mailhe M, Doudier $B$, et al. Hydroxychloroquine and azithromycin as a treatment of COVID-19: Results of an open-label non-randomized clinical trial. Int JAntimicrob Agents. 2020; 56(1):105949.

19. Bacharier LB, Guilbert TW, Mauger DT, Boehmer S, Beigelman A, Fitzpatrick AM et al. Early administration of azithromycin and prevention of severe lower respiratory tract illnesses in preschool children with a history of such illnesses: A randomized clinical trial. JAMA. 2015:314(19); 2034-44.

20. Boltz DA, Rehg JE, McClaren J, Webster RG, Govorkova EA. Oseltamivir prophylactic regimens prevent $\mathrm{H} 5 \mathrm{~N} 1$ influenza morbidity and mortality in a ferret model. J Infect Dis. 2008; 197(9):1315-23.

21. Davies BE. Pharmacokinetics of oseltamivir: An oral antiviral for the treatment and prophylaxis of influenza in diverse populations. J Antimicrob Chemother. 2010; 65(Suppl2): ii5-10.

22. Fowler AA 3rd, Truwit JD, Hite RD, DeWilde C, Priday A, Fisher $B$ et al. Effect of vitamin $\mathbf{C}$ infusion on organ failure and biomarkers of inflammation and vascular injury in patients with sepsis and severe acute respiratory failure: The CITRIS-ALI randomized clinical trial. JAMA.2019:322(13); 1261-70.
23. Carr AC, Rosengrave PC, Bayer S, Chambers S, Mehrtens J, Shaw GM. et al. Hypovitaminosis C and vitamin $\mathbf{C}$ deficiency in critically ill patients despite recommended enteral and parenteral intakes. Crit Care. 2017; $21(1): 300$.

24. Charoenngam N, Holick MF. Immunologic effects of vitamin D on human health and disease. Nutrients. 2020; $12(7): 2097$.

25. Grant WB, Giovannucci E. The possible roles of solar ultraviolet-B radiation and vitamin $D$ in reducing case-fatality rates from the 1918-1919 influenza pandemic in the United States. Dermato endocrinol. 2009; 1(4):215-9.

26. Lemire JM, Adams JS, Sakai R, Jordan SC. 1 alpha, 25-dihydroxyvitamin D3 suppresses proliferation and immunoglobulin production by normal human peripheral blood mononuclear cells. J Clin Invest.1984; 74(2):657-61.

27. Chen S, Sims GP, Chen XX, Gu YY, Chen S, Lipsky PE Modulatory effects of 1,25-dihydroxyvitamin D3 on human B cell differentiation. J Immunol.2007; 179(3):1634-47.

28. Oran DP, Topol EJ. Prevalence of Asymptomatic SARS-CoV-2 Infection: A Narrative Review. Ann Intern Med. 2020; 173(5):362-7.

\begin{tabular}{|c|c|c|c|}
\hline \multicolumn{4}{|c|}{ AUTHORSHIP AND CONTRIBUTION DECLARATION } \\
\hline Sr. \# & Author(s) Full Name & Contribution to the paper & Author(s) Signature \\
\hline 1 & Asma Naseer Cheema & $\begin{array}{l}\text { Conception, conduction, } \\
\text { Analysis, writing. }\end{array}$ & \\
\hline 3 & Muhammad Younas & Analysis, Review. & \\
\hline 4 & Azra Azra & Data collection, Review. & \\
\hline 5 & Yasmeen Batool & Data collection, Review. & $/$ \\
\hline 6 & Syed Zahid Ali Zaidi & Data collection, Review. & \\
\hline
\end{tabular}

